\title{
An Image Compression Algorithm and Its Analysis on the Basis of Different Masking Tables
}

\author{
*Tabassum Bano ${ }^{1}$, Matiur Rehman ${ }^{2}$ \\ ${ }^{1}$ Department of Electronics and Communication Engineering School of Engineering and Technology Al-Falah \\ University, Dhauj, Faridabad, Haryana, INDIA \\ ${ }^{2}$ Department of Electronics and Communication Engineering School of Engineering and Technology Al-Falah \\ University, Dhauj, Faridabad, Haryana, INDIA \\ Corresponding Author: Tabassum Bano
}

\begin{abstract}
Image compression algorithms are used to reduce the redundancies in the representation of the data so that the data storage requirements and the communication cost can be reduced. Different algorithms have been developed to reduce the size of an image like JPEG, PNG, SPIHT, etc. In this paper, we mainly focus on the JPEG image compression algorithm because it is mostly used in the Internet and Multimedia based applications. In JPEG, quantization tables and masking tables are used as the main source for the compression of the data. Based on our review, we identify that in literature less attention is given to analyze the effect of the masking tables during the implementation of the JPEG algorithms. Therefore, to address this research issue, in this paper, we analyze the effect of the masking tables used during the JPEG image compression algorithms.

Keywords: JPEG; Quantization Tables; DCT; Image Compression; Masking Tables
\end{abstract}

\section{INTRODUCTION}

Image has been considered as the preferred data type for the interaction among the users on the social networking sites and also for the sharing purposes. Images on social networks increase the interactivity of the user by $120 \%$ because it takes lesser time to portray meaning [1]. Images are selfexpressive and it can determine the personality and the interests of the users. In today's environment, users are uploading pictures which are captured thorough high resolution cameras. Uploading the large number of images increases the need of the bandwidth for multimedia communication application. The objective of the image compression algorithm is to compress the image in such a way that after reconstruction the image should be represented in the same as it was in original form. The objective of the image compression algorithms is to reduce the image data so that image can be transferred from source to destination by saving channel bandwidth and the storage capacity. Therefore, it motivates us to work in the area of the JPEG image compression algorithms (ICA).

Broadly speaking, "Joint Photographic Expert group" (JPEG) ICA are divided into two parts, i.e., lossless and lossy. After compression of an image, if the reconstructed image is the exact replica of the original images, as determined by the MSE and PSNR, then the compression algorithms are referred to as the lossless ICA, otherwise it is called lossy ICA
[2]. Lossy ICA provides higher compression ratio and the lossless ICA provides lower compression ratio. In lossless ICA, the quality of an image may be compromised but in case of the lossless ICA the visual quality of the image is almost same as the original image [3].

Different algorithms [1, 4] have been developed for the still image coding like "Joint Photographic Expert Group" (JPEG), "Portable Network Graphics" (PNG), "Set Partitioning in Hierarchical Trees" (SPIHT), JPEG 2000, JPEG-LS, "Motion Picture Expert Group-4 Visual Texture Coding" (MPEG-4 VTC). In JPEG algorithm, there are following steps: (i) to divide the image into 8X8 blocks (ii) Discrete Cosine Transform (DST), (iii) Quantization Table, (iv) Zigzag sequence, (v) Entropy coding, (vi) and Huffman Coding. Among these steps, quantization plays an important role during image compression to discard that information which is visually insignificant. Apart from this, there are some other algorithms that use the masking tables during the image compression. Based on our review, we identify that in literature less attention is given to analyze the effect of the masking tables during the implementation of the JPEG algorithms. Therefore, to address this research issue, in this paper, we propose an algorithm for the image compression and analyze the results after implementation using MATrix LABoratory, i.e., MATLAB. 
The remaining part of this paper is organized as follows: The related work in the area of image compression algorithms based on JPEG is given in section II. A brief introduction about the JPEG algorithm is given in section III. Experimental work is carried out in section IV. In this section, we also implement the JPEG algorithm using MATLAB and analyze the results on different masking tables. Finally, conclusions and the future work are given in section $\mathrm{V}$.

\section{RELATED WORK}

The objective of this section is to present the related work in the area of JPEG algorithms [7, 11, 12, 14, 15, 17]. In 2002, Santa-Cruz et al. [1] compared the performance evaluation of the JPEG 2000 with the JPEG-LS, MPEG-4 VTC, JPEG, PNG, and SPIHT algorithms. As a result, authors found that "JPEG 2000 performs well in terms of its ability to efficiently deal with various type of the images". Skodras et al. [4] presented the structure of the JPEG 2000 algorithm and also discuss their performance. In 2011, Sun et al. [5] analyze the JPEG standard in "Wireless Multimedia Sensor Networks" (WMSN). The authors proposed a low energy ICA that is based on one of the research area of the WMSN.

In other studies, Ameer and Basir [6] described a simple method to compress the image thorough plane fitting with inter-block prediction. ZainEldin et al. [8] presented a survey based on the ICA in WMSN. Sau et al. [9] apply the block truncation coding using the Clifford algebra for the compression of digital images. In 2012, Devaraj et al. [10] compare different types of the image compression technique using the "Computer Vision and Image Processing" (CVIP) tools based on the following criteria like "Compression Ratio"(CR), "Peak Signal to Noise Ratio" (PSNR), and "Root Mean Square Error" (RMSE). Shukla et al. [13] presented the survey on lossless comparison methods. In their study, two types of the image compression methods are used for the survey, i.e., "prediction based method" and the "transform based method".

Based on our review, we identify that researchers are devoting more time to proposed the new algorithms for the image compression which given more $\mathrm{CR}$ and maintain the quality of the image as good as possible. In this line of research, Kumar and Singh [14] proposed a method for the image compression for minimizing the number of bits for storing an image into disk. In another study, Patil and Prajapati [16] proposed an ICA without decreasing the quality of the image. In our work, we proposed an algorithm for image compression and the analysis of the algorithm on the basis of the different masking tables.

\section{PROPOSED ALGORITHM}

In this section, we proposed an algorithm for the lossless image compression, which is an extended version of [16]. In our work, we analyze the proposed algorithm on different masking tables. Proposed method includes the following steps:

Step 1: Input the image for compression

Step 2: Convert the input image into double

Step 3: Compute Discrete Cosine Transform

Step 4: Apply masking tables for quantization

Step 5: Computation of compressed ratio on the basis of the following:

(i) When same masking table is used during the compression of RGB components

(ii) When different masking tables are used during the compression of RGB components

Step 6: To show the image after compression

\section{Step 1: Input the image for compression}

The objective of this step is to input the image that we want to compress. In this "imread" function of MATLAB is used to input the image.

\section{Step 2: Convert the input image into double}

In this step, the input is converted from uint8 to double. For example, if the size of an image $\mathrm{I}$ is $384 \times 512 \times 3=589824$, then after applying the MATLAB command im2double (I) the size of the image I would be 4718592 .

\section{Step 3: Compute Discrete Cosine Transform}

In this step, the DCT of the 8X8 block are computed. To compute the DCT, the DCT command of the MATLAB is used in our method.

\section{Step 4: Apply Masking Tables for Quantization}

In this step, we mask the DCT by using different masking tables. In our work, we apply the same masking tables for different red, green, and blue components of an image

\section{Step 5: Computation of Compressed Ratio}

Compression ratio of an image can be computed under two different situations, i.e., (i) when same masking table is used during the compression for RGB components, and (ii) when different masking tables are used during the compression for RGB components. For example, in Table 1 - Table 10, same masking table is used for RGB components. In another case, different masking tables can be used for different components of the image; and it would be discussed in our future work.

\section{Step 6: To show the image after compression}

The objective of this step is to show the image after compression. 


\section{EXPERIMENTAL WORK}

In this section, we have implemented the proposed algorithm using MATLAB. We have compressed the images on different masking tables, as shown in Table 1-Table 10.

Case 1: In this case we use the Table 1 for the image compression; and the results are shown in Fig. 1.

Table 1: Masking Table used for Image Compression

\begin{tabular}{|l|l|l|l|l|l|l|l|}
\hline 1 & 1 & 1 & 1 & 0 & 0 & 0 & 0 \\
\hline 1 & 1 & 1 & 0 & 0 & 0 & 0 & 0 \\
\hline 1 & 1 & 0 & 0 & 0 & 0 & 0 & 0 \\
\hline 1 & 0 & 0 & 0 & 0 & 0 & 0 & 0 \\
\hline 0 & 0 & 0 & 0 & 0 & 0 & 0 & 0 \\
\hline 0 & 0 & 0 & 0 & 0 & 0 & 0 & 0 \\
\hline 0 & 0 & 0 & 0 & 0 & 0 & 0 & 0 \\
\hline 0 & 0 & 0 & 0 & 0 & 0 & 0 & 0 \\
\hline
\end{tabular}

Case 2: In this case we use the Table 2 for the image compression; and the results are shown in Fig. 2.

Case 3: In this case we use the Table 3 for the image compression; and the results are shown in Fig. 3.

Case 4: In this case we use the Table 4 for the image compression; and the results are shown in Fig. 4.

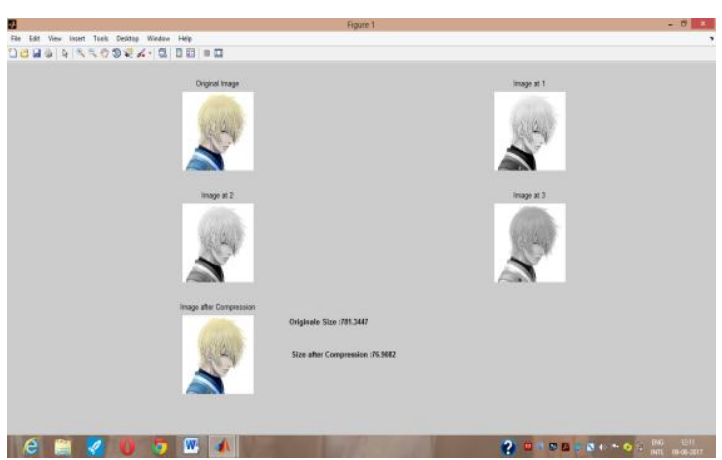

Fig. 1: Image compression based on masking Table 1

Table 2: Masking Table used for Image Compression

\begin{tabular}{|l|l|l|l|l|l|l|l|}
\hline 1 & 1 & 1 & 1 & 1 & 0 & 0 & 0 \\
\hline 1 & 1 & 1 & 0 & 0 & 0 & 0 & 0 \\
\hline 1 & 1 & 0 & 0 & 0 & 0 & 0 & 0 \\
\hline 1 & 0 & 0 & 0 & 0 & 0 & 0 & 0 \\
\hline 1 & 0 & 0 & 0 & 0 & 0 & 0 & 0 \\
\hline 0 & 0 & 0 & 0 & 0 & 0 & 0 & 0 \\
\hline 0 & 0 & 0 & 0 & 0 & 0 & 0 & 0 \\
\hline 0 & 0 & 0 & 0 & 0 & 0 & 0 & 0 \\
\hline
\end{tabular}

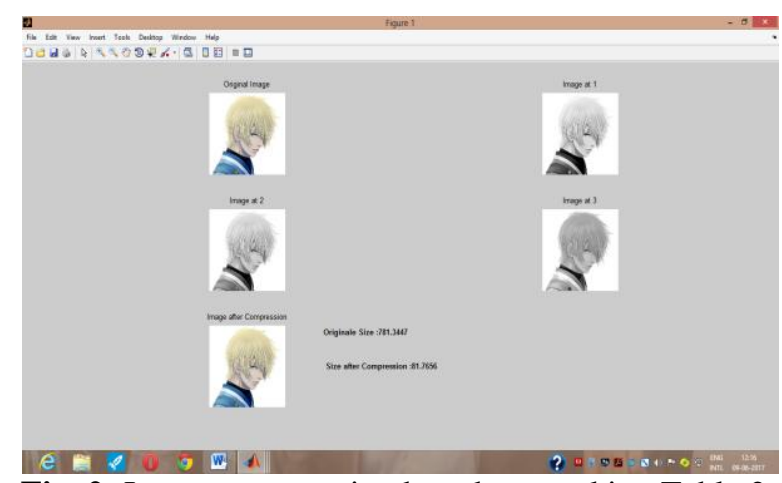

Fig. 2: Image compression based on masking Table 2

Table 3: Masking Table used for Image Compression

\begin{tabular}{|l|l|l|l|l|l|l|l|}
\hline 1 & 1 & 1 & 1 & 1 & 1 & 0 & 0 \\
\hline 1 & 1 & 1 & 0 & 0 & 0 & 0 & 0 \\
\hline 1 & 1 & 0 & 0 & 0 & 0 & 0 & 0 \\
\hline 1 & 0 & 0 & 0 & 0 & 0 & 0 & 0 \\
\hline 1 & 0 & 0 & 0 & 0 & 0 & 0 & 0 \\
\hline 1 & 0 & 0 & 0 & 0 & 0 & 0 & 0 \\
\hline 0 & 0 & 0 & 0 & 0 & 0 & 0 & 0 \\
\hline 0 & 0 & 0 & 0 & 0 & 0 & 0 & 0 \\
\hline
\end{tabular}

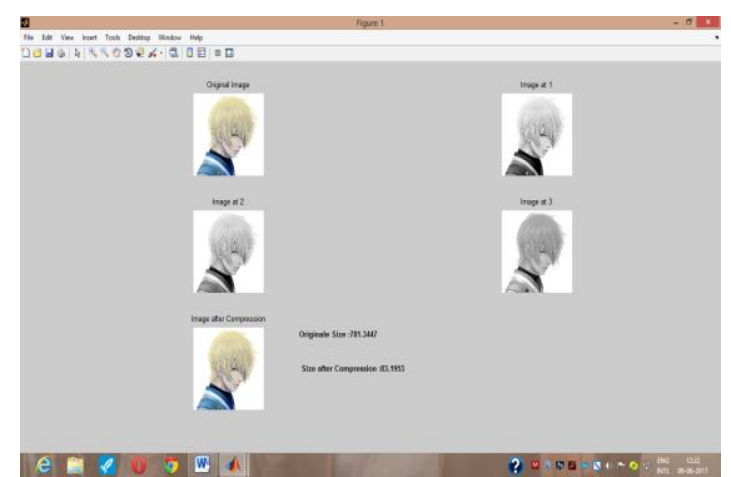

Fig. 3: Image compression based on masking Table 3

Table 4: Masking Table used for Image Compression

\begin{tabular}{|l|l|l|l|l|l|l|l|}
\hline 1 & 1 & 1 & 1 & 1 & 1 & 1 & 0 \\
\hline 1 & 1 & 1 & 0 & 0 & 0 & 0 & 0 \\
\hline 1 & 1 & 0 & 0 & 0 & 0 & 0 & 0 \\
\hline 1 & 0 & 0 & 0 & 0 & 0 & 0 & 0 \\
\hline 1 & 0 & 0 & 0 & 0 & 0 & 0 & 0 \\
\hline 1 & 0 & 0 & 0 & 0 & 0 & 0 & 0 \\
\hline 1 & 0 & 0 & 0 & 0 & 0 & 0 & 0 \\
\hline 0 & 0 & 0 & 0 & 0 & 0 & 0 & 0 \\
\hline
\end{tabular}

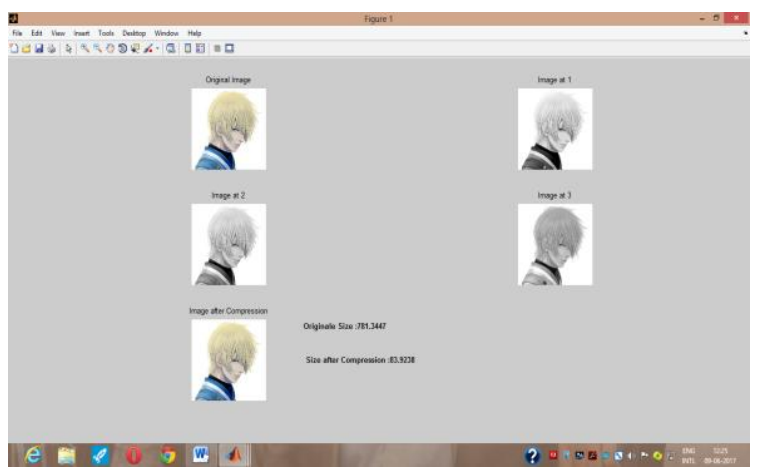

Fig. 4: Image compression based on masking Table 4 
Similarly, we have compressed the image on the following masking tables, i.e., Table 5- Table 10; and the results after the compression are summarized in Table 11.

Table 5: Masking Table used for Image Compression

\begin{tabular}{|l|l|l|l|l|l|l|l|}
\hline 1 & 1 & 1 & 1 & 1 & 1 & 1 & 1 \\
\hline 1 & 1 & 1 & 0 & 0 & 0 & 0 & 0 \\
\hline 1 & 1 & 0 & 0 & 0 & 0 & 0 & 0 \\
\hline 1 & 0 & 0 & 0 & 0 & 0 & 0 & 0 \\
\hline 1 & 0 & 0 & 0 & 0 & 0 & 0 & 0 \\
\hline 1 & 0 & 0 & 0 & 0 & 0 & 0 & 0 \\
\hline 1 & 0 & 0 & 0 & 0 & 0 & 0 & 0 \\
\hline 1 & 0 & 0 & 0 & 0 & 0 & 0 & 0 \\
\hline
\end{tabular}

Table 6: Masking Table used for Image Compression

\begin{tabular}{|l|l|l|l|l|l|l|l|}
\hline 1 & 1 & 1 & 1 & 1 & 1 & 1 & 1 \\
\hline 1 & 1 & 1 & 1 & 0 & 0 & 0 & 0 \\
\hline 1 & 1 & 0 & 0 & 0 & 0 & 0 & 0 \\
\hline 1 & 1 & 0 & 0 & 0 & 0 & 0 & 0 \\
\hline 1 & 0 & 0 & 0 & 0 & 0 & 0 & 0 \\
\hline 1 & 0 & 0 & 0 & 0 & 0 & 0 & 0 \\
\hline 1 & 0 & 0 & 0 & 0 & 0 & 0 & 0 \\
\hline 1 & 0 & 0 & 0 & 0 & 0 & 0 & 0 \\
\hline
\end{tabular}

Table 7: Masking Table used for Image Compression

\begin{tabular}{|l|l|l|l|l|l|l|l|}
\hline 1 & 1 & 1 & 1 & 1 & 1 & 1 & 1 \\
\hline 1 & 1 & 1 & 1 & 1 & 0 & 0 & 0 \\
\hline 1 & 1 & 0 & 0 & 0 & 0 & 0 & 0 \\
\hline 1 & 1 & 0 & 0 & 0 & 0 & 0 & 0 \\
\hline 1 & 1 & 0 & 0 & 0 & 0 & 0 & 0 \\
\hline 1 & 0 & 0 & 0 & 0 & 0 & 0 & 0 \\
\hline 1 & 0 & 0 & 0 & 0 & 0 & 0 & 0 \\
\hline 1 & 0 & 0 & 0 & 0 & 0 & 0 & 0 \\
\hline
\end{tabular}

Table 8: Masking Table used for Image Compression

\begin{tabular}{|l|l|l|l|l|l|l|l|}
\hline 1 & 1 & 1 & 1 & 1 & 1 & 1 & 1 \\
\hline 1 & 1 & 1 & 1 & 1 & 1 & 0 & 0 \\
\hline 1 & 1 & 0 & 0 & 0 & 0 & 0 & 0 \\
\hline 1 & 1 & 0 & 0 & 0 & 0 & 0 & 0 \\
\hline 1 & 1 & 0 & 0 & 0 & 0 & 0 & 0 \\
\hline 1 & 1 & 0 & 0 & 0 & 0 & 0 & 0 \\
\hline 1 & 0 & 0 & 0 & 0 & 0 & 0 & 0 \\
\hline 1 & 0 & 0 & 0 & 0 & 0 & 0 & 0 \\
\hline
\end{tabular}

Table 9: Masking Table used for Image Compression

\begin{tabular}{|l|l|l|l|l|l|l|l|}
\hline 1 & 1 & 1 & 1 & 1 & 1 & 1 & 1 \\
\hline 1 & 1 & 1 & 1 & 1 & 1 & 1 & 0 \\
\hline 1 & 1 & 0 & 0 & 0 & 0 & 0 & 0 \\
\hline 1 & 1 & 0 & 0 & 0 & 0 & 0 & 0 \\
\hline 1 & 1 & 0 & 0 & 0 & 0 & 0 & 0 \\
\hline 1 & 1 & 0 & 0 & 0 & 0 & 0 & 0 \\
\hline 1 & 1 & 0 & 0 & 0 & 0 & 0 & 0 \\
\hline 1 & 0 & 0 & 0 & 0 & 0 & 0 & 0 \\
\hline
\end{tabular}

Table 10: Masking Table used for Image Compression

\begin{tabular}{|l|l|l|l|l|l|l|l|}
\hline 1 & 1 & 1 & 1 & 1 & 1 & 1 & 1 \\
\hline 1 & 1 & 1 & 1 & 1 & 1 & 1 & 1 \\
\hline 1 & 1 & 0 & 0 & 0 & 0 & 0 & 0 \\
\hline 1 & 1 & 0 & 0 & 0 & 0 & 0 & 0 \\
\hline 1 & 1 & 0 & 0 & 0 & 0 & 0 & 0 \\
\hline 1 & 1 & 0 & 0 & 0 & 0 & 0 & 0 \\
\hline 1 & 1 & 0 & 0 & 0 & 0 & 0 & 0 \\
\hline 1 & 1 & 0 & 0 & 0 & 0 & 0 & 0 \\
\hline
\end{tabular}

Table 11: Size of the compressed images based on different masking tables

\begin{tabular}{|c|l|l|l|}
\hline $\begin{array}{c}\text { S. } \\
\text { No. }\end{array}$ & $\begin{array}{l}\text { Masking } \\
\text { Table }\end{array}$ & $\begin{array}{l}\text { Size of } \\
\text { original } \\
\text { image in } \\
(\text { KB })\end{array}$ & $\begin{array}{l}\text { Size of the } \\
\text { image after } \\
\text { compression in } \\
(\text { KB })\end{array}$ \\
\hline 1 & Table 1 & 781.3447 & 76.9082 \\
\hline 2 & Table 2 & 781.3447 & 81.7656 \\
\hline 3 & Table 3 & 781.3447 & 83.1953 \\
\hline 4 & Table 4 & 781.3447 & 83.9238 \\
\hline 5 & Table 5 & 781.3447 & 84.165 \\
\hline 6 & Table 6 & 781.3447 & 87.747 \\
\hline 7 & Table 7 & 781.3447 & 90.1895 \\
\hline 8 & Table 8 & 781.3447 & 90.9004 \\
\hline 9 & Table 9 & 781.3447 & 91.2529 \\
\hline 10 & Table & 781.3447 & 91.3975 \\
\hline
\end{tabular}

On the basis of the results of the Table 11, we identify that, as we are going to increase the number of one's in some particular direction, we are getting different compression ratio. This compression ratio is increasing by changing the number of one's in the masking tables. Therefore, at the time of compression of the images, it is important to find out that which masking table pattern would be useful for the compression of the image.

Apart from the above masking tables, on the basis of our experimental work, we observe that when we have only single one in the masking table at the zero-zero location of the making table, the size of the compression ratio gets reduced; and it comes out to be $33.8345 \mathrm{~KB}$. It means that, we cannot compress the image beyond $33.8345 \mathrm{~KB}$ because here we are not masking the DC coefficient of the masking table, while all the AC coefficients are masked with zeros.

\section{CONCLUSIONS AND FUTURE WORK}

This paper presents an algorithm for the compression of the digital images. Proposed algorithm includes the following steps: (i) input the image for compression, (ii) convert the input image into double, (iii) compute DCT, (iv) apply masking tables, (v) computation of compressed ratio on the basis of the following: (a) When same masking table is used during the compression for RGB components, (b) 
When different masking tables are used during the compression for RGB components, (vi) to show the image after compression. We have compressed the image on ten different masking tables. On the basis of our analysis, we identify that size of the compression ratio depends on the type of the making table used during the image compression. In our work, we mainly focus on the same making table which is used

\section{REFERENCES}

[1] Santa-Cruz D., Grosbois R., and Ebrahimi T., "JPEG 2000 Performance Evaluation and Assessment", Signal Processing: Image Communication, Vol. 17, pp. 113-130, 2002.

[2] Marcus M, "JPEG Image Compression", Technical Report, pp. 1-5, 2014

[3] Rehman M., Sharif M. and Raza M., "Image Compression: A Survey", Research Journal of Applied Science, Engineering \& Technology 7I4): 656-672, 2014.

[4] Skordas A. N., Christopoulos A. C., Ebrahimi T.,"JPEG2000: The upcoming still image compression standard", Elsevier, Pattern Recognition Letters (2001) 13371345.

[5] Sun E., Shen X. and Chen H., "A Low Energy Image compression and Transmission in Wireless Multimedia Sensor Networks", Elsevier, Procedia Engineering 15 (2011) 3604-3610.

[6] Ameer S., Basir O., "Image Compression using plane fitting with inter-block prediction", Image and vision computing 27 (2009) 385-390.

[7] Rabbani M., Joshi R.," An Overview of the JPEG 2000 Still Image Compression Standard", Signal Processing and Image Communication 17 (2002) 3-48.

[8] ZainEldin H., Elhosseini A. M., Ali A. H., "Image Compression algorithms in wireless multimedia sensor networks: A Survey", Ains Shams Engineering Journal (2015) 6, 481-490.

[9] Sau K., Basak K. R. and Chanda A., "Image Compression based on Block Truncation coding using Clifford Algebra", Science Direct, Procedia Technology 10 (2013) 699706.

[10] Devaraj J. S., Ezra K., and Kasaraneni K. K., "Survey on Image Compression Techniques: in all the components of the image, i.e., red, green, and blue components. In future, we will work on the following issues: (a) when the different masking tables are used for different components of the images (b) to compress the images of different types like gray images, black and white images, etc.

Using CVIP Tools" CCSIT 2012, Part III, LNIST 86, pp. 300-309, 2012.

[11] S. REmya, V. A. Rasheed Dilshad, Wavelet Based Compression Techniques: A Survey, V.V. Das and J. Stephan (EDs.): CNC 2012, LNICST 108, pp.394-397, 2012.

[12] Mammeri A., Hadjou B., and Khoumsi A., "A Survey of Image compression Algorithms for Visual Sensor Networks", International Scholarly Research Network ISRN Sensor Networks Volume 2012, Article ID 760320, 19 pages.

[13] Shukla J., Alwani M.,and Tiwari k. A., "A Survey On Lossless Image Compression Method", IEEE $2^{\text {nd }}$ International Conference on computer Engineering and Technology, Volume 6, pp $136-141$.

[14] Kumar A., Singh P., "An Image Compression Algorithm for Gray Scale Image", IEEE, International Conference on Emerging Trends in Networks and Computer Communications (ETNCC), Udaipur, 2011.

[15] Kavitha S., and Anandhi J. R.,"A Survey of image compression methods for low depthof -field images and image sequences", Multimedia Tools and Applications, September (2015), Volume 74, Issue 18, pp 7493-7956.

[16] Patil G. L. and Ravindra Prajapati, "Lossless Image Compression Technique for Personal Digital Assistant", International Journal for Scientific Research \& development, Vol 3, issue 02,2015, ISSN No:2321-0613.

[17] Khowaja A. S. and Ismaili A. I. "'Image Compression Using Moving Average Histogram and RBF Network", Mehran University Research Journal of Engineering and Technology, Volume 34, No. 4, October, 2015, ISSSN No:0254-7821.

International Journal of Engineering Research and Applications (IJERA) is UGC approved Journal with S1. No. 4525, Journal no. 47088. Indexed in Cross Ref, Index Copernicus (ICV 80.82), NASA, Ads, Researcher Id Thomson Reuters, DOAJ.

Tabassum Bano. "An Image Compression Algorithm and Its Analysis on the Basis of Different Masking Tables." International Journal of Engineering Research and Applications (IJERA) 7.7 (2017): 06-10. 